\title{
Gravitational sedimentation of cloud of solid spherical particles at small Reynolds numbers
}

\author{
Vladimir Arkhipov ${ }^{1,2}$ and Anna Usanina ${ }^{3}$ \\ ${ }^{1}$ Scientific Research Institute of Applied Mathematics and Mechanics of Tomsk State University, \\ Tomsk, Russia \\ ${ }^{2}$ Institute for Problems of Chemical and Energetic Technologies SB RAS, Biysk, Russia \\ ${ }^{3}$ Tomsk State University, Tomsk, Russia
}

\begin{abstract}
The experimental results of study of gravitational sedimentation of highlyconcentrated systems of solid spherical particles at small Reynolds numbers $\operatorname{Re}<1$ are presented. Empirical equation for drag coefficient of the particle assembly has been obtained. The influence of initial particle concentration in the cloud on its dynamics and velocity has been analysed.
\end{abstract}

\section{Introduction}

The problem of behavior of the system of solid spherical particles at their gravitational sedimentation is one of the fundamental problems of classical hydrodynamics and has an obvious interest at solving the different practical tasks [1]. In particular, in order to provide the effective water basin cleaning from coal particles it is necessary to know the value of optimal concentration of settled particles [2]. At solving the formation of precipitation problem and estimating the ecological consequences at emergency and stage separation of launch rocket [3] it is necessary to have an idea of overall picture of particle group motion in dispersion medium and laws of change of particle system velocity in dependence on sedimentation height and initial parameters (initial concentration and size of particles cloud).

Experimental studies of the process of gravitational sedimentation of highly-concentrated aerosol systems were performed by different authors [4-8]. However, the integration of these results is difficult due to different experiment conditions and methods of processing of the results. In these works the processing of the experimental results in the form of empirical equation for drag coefficient of particles cloud was not performed.

In the present work, the results of experimental study of sedimentation dynamics and drag coefficient for the cloud of solid spherical particles at small Reynolds numbers are presented.

\section{Experimental setup and study technique}

The study was carried out on a setup consisting of a vertically installed transparent cell with planeparallel walls, which was filled with glycerin, a system for particles emission, and a system for

This is an Open Access article distributed under the terms of the Creative Commons Attribution License 4.0, which permits unrestricted use, distribution, and reproduction in any medium, provided the original work is properly cited. 


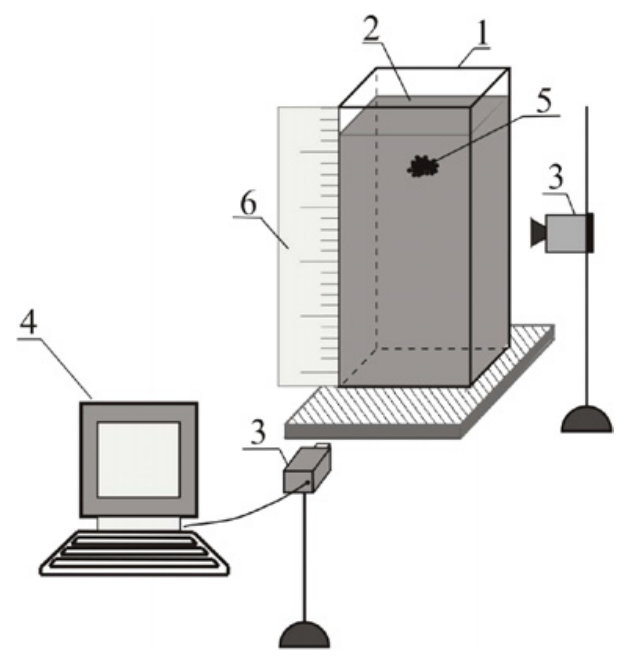

Figure 1. Block diagram of the experimental setup: 1 - cell; 2 - glycerin; 3 - video cameras; 4 - computer; 5 particle cloud; 6 - scale rule.
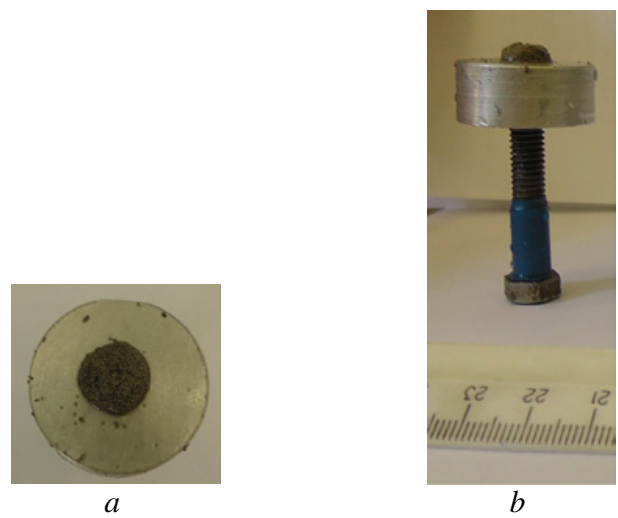

Figure 2. Construction for creating the particle cloud: $a$-overhead view; $b$ - side view.

visualizing the process of gravitational sedimentation of a highly-concentrated system of solid spherical particles. Steel spheres in diameter of $d=0.2 \mathrm{~mm}$ and $d=0.7 \mathrm{~mm}$ were used in the experiments. A block diagram and photograph of the experimental setup are presented in Fig. 1. The cell, of dimension $300 \times 300 \times 900 \mathrm{~mm}$ (of volume 81 liters), was manufactured from optical glass of thickness $15 \mathrm{~mm}$.

In order to create a highly-concentrated cloud of uniformly distributed solid spherical particles it was used the following method. On electronic weighing machine the fixed mass of the particles was weighed. Then this mass of particles was moistened by glycerin and was located on a plain horizontal aluminum substrate of diameter $3 \mathrm{~cm}$ (Fig. 2). Due to a large value of coefficient of dynamic viscosity of glycerin and a small particles size this layer of particles was not decomposed. Then the substrate with this layer of particles was moved down in the cell with glycerin. When the particles are contacted with the glycerin their uniform emission occurs and the uniform cloud of particles is formed. In the experiments varying the layer thickness of the particles on the substrate the clouds of particles with different initial concentration were obtained. 
The visualization system incorporated light sources (two $18 \mathrm{~W}$ lamps), a Panasonic HDC-SD60 digital video camera, and a Citius $\mathrm{C} 100$ high-speed video camera. Twoangle video recording of the process under study enabled to improve precision and to ensure monitoring of the change in the parameters of gravitational sedimentation of the cloud of particles throughout the cell height. The first camera was used for recording of the overall picture and motion dynamics of the cloud of particles; the recording field was $5 \times 5 \mathrm{~cm}$ with a two fold magnification. The second camera recorded the velocity of sedimentation of particle system throughout the motion trajectory. Video recording was performed with a spatial resolution of $576 \times 1024$ pixels with a rate of 100 frames per second and an exposure time of $(1.0 \div 2.0) \mathrm{ms}$. A scale rule with a scale factor of $1 \mathrm{~mm}$ calibrated with allowance for the optical distortion by cell walls was used to monitor the distance covered by the cloud.

\section{Measured parameters}

During the experiments, measurements of all basic parameters of the process were carried out: the density $\rho_{l}$ and the coefficient of dynamic viscosity of glycerin $\mu_{l}$, the initial concentration of the cloud $C_{V 0}$, the diameter of the cloud $D$, the distance covered by it $h(t)$, and the sedimentation velocity $u(t)$. Physical properties of glycerin were measured before and after each experiment.

The density of glycerin was equal to $\rho_{l}=1260 \mathrm{~kg} / \mathrm{m}^{3}$ and was measured by an aerometer with a relative error $\delta \rho_{l}=0.1 \%$.

The coefficient of dynamic viscosity of glycerin was ranged within $\mu_{l}=(1.12 \pm 1.17) \mathrm{Pa} \cdot \mathrm{s}$ and was determined from the measured velocity of stationary settling $u_{p}$ of a steel sphere of diameter $D_{p}=1.3 \mathrm{~mm}$ in the Stokesian regime $(\operatorname{Re}<1)$ :

$$
\mu_{l}=\frac{g D_{p}^{2}\left(\rho_{p}-\rho_{l}\right)}{18 u_{p}},
$$

where $\rho_{p}$ is the density of the sphere's material.

The diameter of the cloud was determined by the formula

$$
D_{s}=\frac{D_{1}^{c p}+D_{2}^{c p}}{2}
$$

where $D_{1}^{c p}, D_{2}^{c p}$ is the average value of midsection of the cloud in horizontal and vertical directions, respectively, obtained from twoangle video recording. The value of diameter of the cloud was equal to $D_{s}=(0.5 \div 1.78) \mathrm{cm}$.

The initial concentration of the cloud was determined by means of the equation

$$
C_{V 0}=\frac{m}{m_{1}} \frac{d^{3}}{D_{s}^{3}},
$$

where $m$ is the mass of particles in the cloud; $m_{1}$ is the mass of a single particle; $d$ is the diameter of a single particle. In the experiments the value of the initial concentration was varied within $C_{V 0}=$ $0.032 \div 0.47$.

The mass of a single particle was computed from the formula

$$
m_{1}=\frac{M}{N}
$$

where $N=200$ is the number of particles in the cloud; $M$ is the mass of $N$ particles.

The distance covered by the cloud and the velocity were determined by frame-frame processing of results of the high-speed recording. The sedimentation velocity of the particle cloud at a certain 


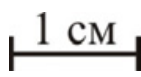

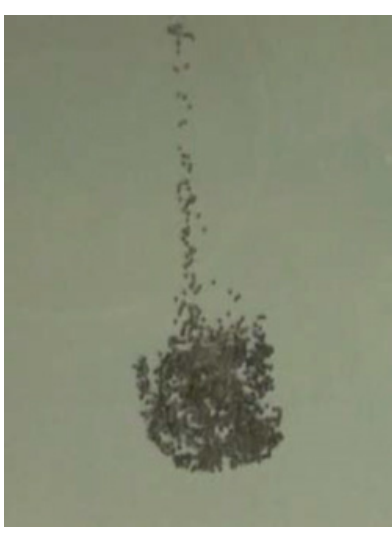

$a$

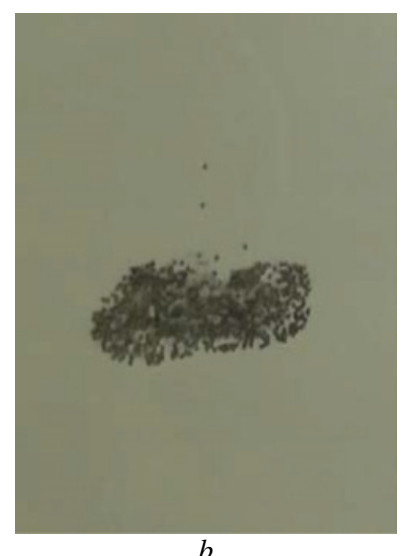

$b$

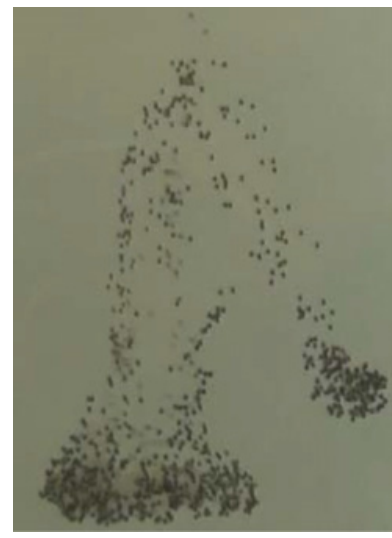

Figure 3. The stages of the process of gravitational sedimentation of solid spherical particles $(d=0.2 \mathrm{~mm}$; $\left.\mu_{l}=1.17 \mathrm{~Pa} \cdot \mathrm{s} ; D_{s}=1.12 \mathrm{ni} ; N_{V 0}=0.054\right): a$-spheroid formation; $b$-spheroid motion; $c$ - spheroid breakup.

height $h_{i}$ (where $i$ is the frame number) was computed from the formula

$$
u\left(h_{i}\right)=\frac{h_{i+1}-h_{i-1}}{\Delta t_{i}}, \quad i=1,2, \ldots, n,
$$

where $h_{i-1}, h_{i+1}$ is the distance covered by the cloud on the $i-1$ and $i+1$ frames, respectively; $\Delta t_{i}$ is the time interval between $i-1$ and $i+1$ frames; $n$ is the number of frames. The distance $h_{i}$ was measured with the use of computer program CorelDRAW. The error of measurement of $h_{i}$ associated with the distortion image and the resolving power of the video was $\pm 0.2 \mathrm{~mm}$.

The average velocity of the particle cloud was computed using a formula

$$
\bar{u}=\frac{h}{\Delta t},
$$

where $h$ is the distance covered by the cloud; $\Delta t$ is the time of covering the distance $h$ by the cloud. The value of the average sedimentation velocity of particle cloud was varied within $\bar{u}=(0.6 \div 7.8) \mathrm{cm} / \mathrm{s}$ in the experiments.

\section{Experimental results}

In the range of Reynolds numbers $\operatorname{Re}=7 \cdot 10^{-2} \div 1$, it was found that the process of particle system sedimentation can be divided into three stages: the stage of formation, motion and breakup of spheroid (Fig. 3).

On the first stage the formation of spheroidal cloud occurs accompanied by circulation of peripheral particles in the opposite direction to cloud motion. The time of spheroid formation $t$ depends on the initial mass and concentration of the particle cloud. For the present experiments this time was varied within $t=(1 \div 4) \mathrm{s}$.

On the second stage the cloud has the spheroidal shape and moves without sacrificing the circulation of the peripheral particles. Gradual expansion of the cloud in two diametral direction of the spheroid is taken place.

On the third, final, stage the cloud falls into $2 \div 3$ fragments. The breakup of the cloud occurs from the centre of mass so that the system becomes cupola-shaped. In each secondary fragments the circulating 


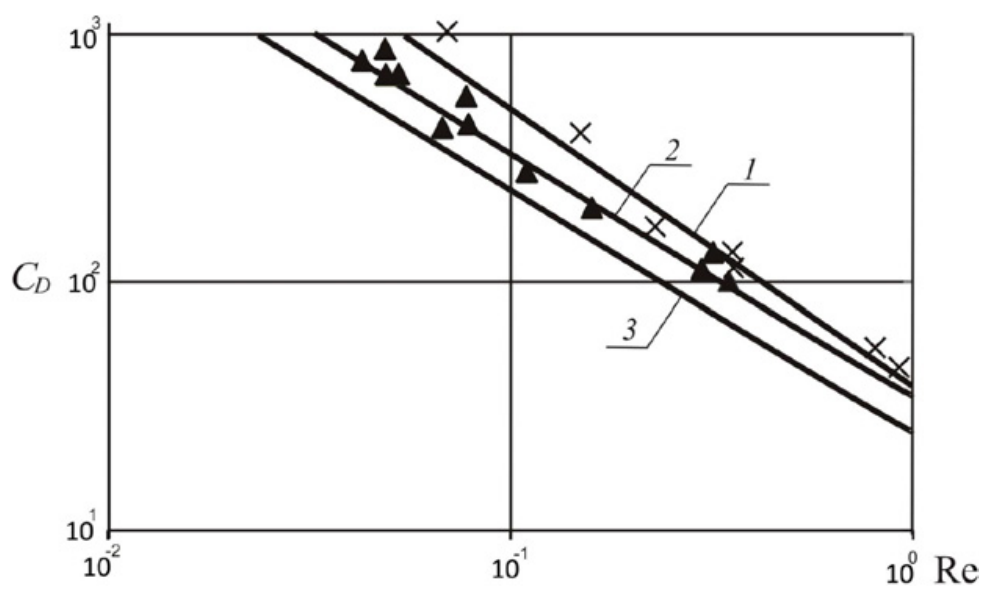

Figure 4. Experimental dependence of the drag coefficient of system particle on Reynolds number: $1-C_{V 0}=$ $0.035 ; 2-C_{V 0}=0.24 ; 3-$ Stokes's equation $\left(C_{D}=24 / \mathrm{Re}\right)$.

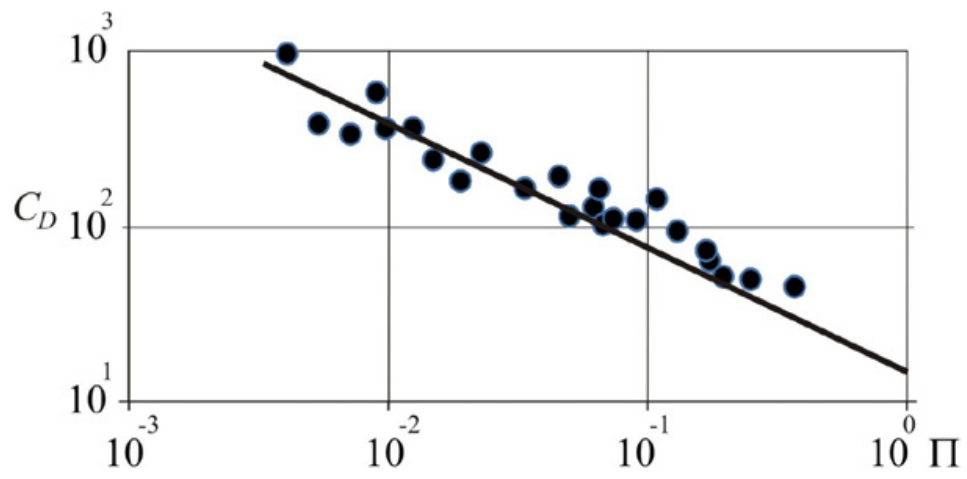

Figure 5. Experimental dependence of the drag coefficient for particle cloud on complex Re. $C_{V 0}$.

motion of the peripheral particles is also observed. The process of breakup of the system is cyclical and the breakup of secondary clouds lasts as long as the system moves with the velocity of a single particle of the cloud.

On the base of the experimental results the computation of experimental value of drag coefficient of particle system was carried out using the formula

$$
C_{D}=\frac{4}{3} \frac{g D_{s} C_{V 0}}{\rho_{l} \bar{u}^{2}}\left(\rho-\rho_{l}\right),
$$

where $g=9.80665 \mathrm{~m} / \mathrm{s}^{2}$ is the gravitational acceleration; $\rho$ is the density of particle's material.

Figure 4 shows the dependence of the drag coefficient of particle system on Reynolds number at different values of the initial concentration.

The dependences processing in the Fig. 4 showed that while the initial concentration increases the dependence for particle cloud $C_{D}(\mathrm{Re})$ tends to Stokes's equation for a single solid spherical particle $\left(C_{D}=24 / \mathrm{Re}\right)$. The Fig. 4 indicates that in the studied range of Reynolds number at increasing the initial concentration the drag coefficient for the cloud of particles of the same size decreases and the sedimentation velocity, respectively, grows. 
Analysis of the experimental results enables to receive the generalized empirical formula for drag coefficient for the particle cloud in dependence on dimensionless complex $\Pi=\operatorname{Re} \cdot C_{V 0}$, where $\operatorname{Re}=D_{s} \bar{u} \rho_{l} / \mu_{l}$.

In the Fig. 5 the experimental results processed with using the dependence $C_{D}=f(\Pi)$ are presented. Approximation of these results (full line in the Fig. 5) is described by the equation

$$
C_{D}=\frac{14.6}{\left(\operatorname{Re} \cdot C_{V 0}\right)^{0.7}}
$$

This equation takes into account the influence of both the initial concentration of the particles and the regime of the cloud motion on the drag coefficient and has higher value of correlation factor $\left(R^{2}=0.81\right)$ as compared to other approximating formulas.

\section{Conclusion}

According to the experimental results the following conclusions can be made.

- New experimental results on gravitational sedimentation of a highly-concentrated system of solid spherical particles at the range of Reynolds number $\mathrm{Re}=7 \cdot 10^{-2} \div 1$ have been obtained.

- It has been shown that the process of sedimentation of the particle cloud can be divided into three stages: formation, motion and breakup of spheroidal cloud.

- The generalized empirical formula for the drag coefficient of particle cloud in dependence on dimensionless complex $\Pi=\mathrm{Re} \cdot C_{V 0}$ has been received.

- It has been shown that while the complex $\Pi$ increases within the range of $3 \cdot 10^{-3} \div 0.4$ the value of the drag coefficient decreases in 20 times $(1013 \div 45)$.

- The obtained experimental results can be used for improvement of existing physical-mathematical models for evolution of liquid-drop aerosol in atmosphere.

This work was carried out with the financial support from the grant of the President of the Russian Federation for state support of young Russian scientists, candidates of sciences (No. IE-1259.2013.1) and Ministry of Education and Science of the Russian Federation No. 2014/223 (project code 1567).

\section{References}

[1] B. Brounshtein, G. Fishbein, Hydrodynamics, mass- and heat transfer in dispersed systems (Leningrad, Chemistry, 1977)

[2] I. Vasenin, K. Elkin, Russian Physics Journal, 3, 109 (1999)

[3] V. Arkhipov, A. Berezikov, A. Tkachenko, A. Usanina, O. Yakimovich, Aviakosmicheskoe priborostroenie, 5, 33 (2011)

[4] V. Horguani, Izvestiya of the Academy of Sciences of the USSR. Atmospheric and Oceanic Physics, 2, 394 (1966)

[5] V. Horguani, Materials of HGI, 13, 97 (1969)

[6] J. Nitsche, G. Batchelor, Journal of Fluid Mechanics, 340, 161 (1997)

[7] G. Machu, W. Meile, L. Nitsche, U. Schaflinger, Journal of Fluid Mechanics, 447, 299 (2001)

[8] B. Metzger, M. Ekiel-Jezewska, E. Guazzelli, Physics of Fluids, 18, 038104 (2006) 